\title{
CrystEngComm
}

Check for updates

Cite this: CrystEngComm, 2021, 23, 5367

Received 29th January 2021,

Accepted 3rd July 2021

DOI: $10.1039 / \mathrm{d} 1 \mathrm{ce} 00145 \mathrm{k}$

rsc.li/crystengcomm

\section{A bug in enantiomer separation: double salt formation - diastereomeric and double salt structures of 1-cyclohexylethylammonium 2- and 4-chloromandelate $\dagger$}

\author{
Laura Bereczki, (D)*ab Amit Zodge, ${ }^{c}$ Márton Körösi, ${ }^{c}$ Tamás Holczbauer, (D) ad \\ Sourav De, (iD ${ }^{a}$ Edit Székely (iD ${ }^{c}$ and Petra Bombicz (D) ${ }^{a}$
} \begin{abstract}
The number of crystal structures of diastereomeric salt pairs and especially of double salts is limited in the
literature. This work exceptionally presents the structures of two constitutional isomer double salts along
with their related diastereomeric salt pairs, which were successfully crystallized and their structures
elucidated presenting two complete sets of chiral systems. These results provide a deeper insight into chiral
recognition and contribute to the mastery of synthon engineering. The investigated systems are
1-cyclohexylethylammonium 2 -chloromandelate ( $S$ - $S, R-S$, SS-SR) and 1-cyclohexylethylammonium
4 -chloromandelate ( $R-R$, $S$ - $R$, SS-SR). The crystal structures and the thermal properties of all diastereomers
(including the less stable diastereomers) and double salts have been determined and comparatively
analysed. In the crystal of five of the six chiral salts, hydrogen bonded layers are formed with the
participation of the ionic groups and the hydroxyl group of the mandelate anion. In one structure, the
hydrogen bond layers are closed to form tubes. Due to the different position of the chlorine substituent in
the two compound families, the halogen interactions are oriented towards the inside of the hydrogen-
bonded structures or positioned between the layers and establish a relatively strong connection between
them. The two different halogen positions and every possible combinations of configurations in the six
investigated salts provide a quite detailed landscape of the effect of stereochemistry on the solid-state
structure of the salts. The number of crystal structures of diastereomeric salt pairs and especially of double salts is limited in the
literature. This work exceptionally presents the structures of two constitutional isomer double salts along
with their related diastereomeric salt pairs, which were successfully crystallized and their structures
elucidated presenting two complete sets of chiral systems. These results provide a deeper insight into chiral
recognition and contribute to the mastery of synthon engineering. The investigated systems are
1-cyclohexylethylammonium 2 -chloromandelate ( $S$ - $S, R-S$, SS-SR) and 1-cyclohexylethylammonium
4 -chloromandelate ( $R-R$, $S$ - $R$, SS-SR). The crystal structures and the thermal properties of all diastereomers
(including the less stable diastereomers) and double salts have been determined and comparatively
analysed. In the crystal of five of the six chiral salts, hydrogen bonded layers are formed with the
participation of the ionic groups and the hydroxyl group of the mandelate anion. In one structure, the
hydrogen bond layers are closed to form tubes. Due to the different position of the chlorine substituent in
the two compound families, the halogen interactions are oriented towards the inside of the hydrogen-
bonded structures or positioned between the layers and establish a relatively strong connection between
them. The two different halogen positions and every possible combinations of configurations in the six
investigated salts provide a quite detailed landscape of the effect of stereochemistry on the solid-state
structure of the salts. The number of crystal structures of diastereomeric salt pairs and especially of double salts is limited in the
literature. This work exceptionally presents the structures of two constitutional isomer double salts along
with their related diastereomeric salt pairs, which were successfully crystallized and their structures
elucidated presenting two complete sets of chiral systems. These results provide a deeper insight into chiral
recognition and contribute to the mastery of synthon engineering. The investigated systems are
1-cyclohexylethylammonium 2 -chloromandelate ( $S$ - $S, R-S$, SS-SR) and 1-cyclohexylethylammonium
4 -chloromandelate ( $R-R$, $S$ - $R$, SS-SR). The crystal structures and the thermal properties of all diastereomers
(including the less stable diastereomers) and double salts have been determined and comparatively
analysed. In the crystal of five of the six chiral salts, hydrogen bonded layers are formed with the
participation of the ionic groups and the hydroxyl group of the mandelate anion. In one structure, the
hydrogen bond layers are closed to form tubes. Due to the different position of the chlorine substituent in
the two compound families, the halogen interactions are oriented towards the inside of the hydrogen-
bonded structures or positioned between the layers and establish a relatively strong connection between
them. The two different halogen positions and every possible combinations of configurations in the six
investigated salts provide a quite detailed landscape of the effect of stereochemistry on the solid-state
structure of the salts. The number of crystal structures of diastereomeric salt pairs and especially of double salts is limited in the
literature. This work exceptionally presents the structures of two constitutional isomer double salts along
with their related diastereomeric salt pairs, which were successfully crystallized and their structures
elucidated presenting two complete sets of chiral systems. These results provide a deeper insight into chiral
recognition and contribute to the mastery of synthon engineering. The investigated systems are
1-cyclohexylethylammonium 2 -chloromandelate ( $S$ - $S, R-S$, SS-SR) and 1-cyclohexylethylammonium
4 -chloromandelate ( $R-R$, $S$ - $R$, SS-SR). The crystal structures and the thermal properties of all diastereomers
(including the less stable diastereomers) and double salts have been determined and comparatively
analysed. In the crystal of five of the six chiral salts, hydrogen bonded layers are formed with the
participation of the ionic groups and the hydroxyl group of the mandelate anion. In one structure, the
hydrogen bond layers are closed to form tubes. Due to the different position of the chlorine substituent in
the two compound families, the halogen interactions are oriented towards the inside of the hydrogen-
bonded structures or positioned between the layers and establish a relatively strong connection between
them. The two different halogen positions and every possible combinations of configurations in the six
investigated salts provide a quite detailed landscape of the effect of stereochemistry on the solid-state
structure of the salts. The number of crystal structures of diastereomeric salt pairs and especially of double salts is limited in the
literature. This work exceptionally presents the structures of two constitutional isomer double salts along
with their related diastereomeric salt pairs, which were successfully crystallized and their structures
elucidated presenting two complete sets of chiral systems. These results provide a deeper insight into chiral
recognition and contribute to the mastery of synthon engineering. The investigated systems are
1-cyclohexylethylammonium 2 -chloromandelate ( $S$ - $S, R-S$, SS-SR) and 1-cyclohexylethylammonium
4 -chloromandelate ( $R-R$, $S$ - $R$, SS-SR). The crystal structures and the thermal properties of all diastereomers
(including the less stable diastereomers) and double salts have been determined and comparatively
analysed. In the crystal of five of the six chiral salts, hydrogen bonded layers are formed with the
participation of the ionic groups and the hydroxyl group of the mandelate anion. In one structure, the
hydrogen bond layers are closed to form tubes. Due to the different position of the chlorine substituent in
the two compound families, the halogen interactions are oriented towards the inside of the hydrogen-
bonded structures or positioned between the layers and establish a relatively strong connection between
them. The two different halogen positions and every possible combinations of configurations in the six
investigated salts provide a quite detailed landscape of the effect of stereochemistry on the solid-state
structure of the salts. The number of crystal structures of diastereomeric salt pairs and especially of double salts is limited in the
literature. This work exceptionally presents the structures of two constitutional isomer double salts along
with their related diastereomeric salt pairs, which were successfully crystallized and their structures
elucidated presenting two complete sets of chiral systems. These results provide a deeper insight into chiral
recognition and contribute to the mastery of synthon engineering. The investigated systems are
1-cyclohexylethylammonium 2 -chloromandelate ( $S$ - $S, R-S$, SS-SR) and 1-cyclohexylethylammonium
4 -chloromandelate ( $R-R$, $S$ - $R$, SS-SR). The crystal structures and the thermal properties of all diastereomers
(including the less stable diastereomers) and double salts have been determined and comparatively
analysed. In the crystal of five of the six chiral salts, hydrogen bonded layers are formed with the
participation of the ionic groups and the hydroxyl group of the mandelate anion. In one structure, the
hydrogen bond layers are closed to form tubes. Due to the different position of the chlorine substituent in
the two compound families, the halogen interactions are oriented towards the inside of the hydrogen-
bonded structures or positioned between the layers and establish a relatively strong connection between
them. The two different halogen positions and every possible combinations of configurations in the six
investigated salts provide a quite detailed landscape of the effect of stereochemistry on the solid-state
structure of the salts. The number of crystal structures of diastereomeric salt pairs and especially of double salts is limited in the
literature. This work exceptionally presents the structures of two constitutional isomer double salts along
with their related diastereomeric salt pairs, which were successfully crystallized and their structures
elucidated presenting two complete sets of chiral systems. These results provide a deeper insight into chiral
recognition and contribute to the mastery of synthon engineering. The investigated systems are
1-cyclohexylethylammonium 2 -chloromandelate ( $S$ - $S, R-S$, SS-SR) and 1-cyclohexylethylammonium
4 -chloromandelate ( $R-R$, $S$ - $R$, SS-SR). The crystal structures and the thermal properties of all diastereomers
(including the less stable diastereomers) and double salts have been determined and comparatively
analysed. In the crystal of five of the six chiral salts, hydrogen bonded layers are formed with the
participation of the ionic groups and the hydroxyl group of the mandelate anion. In one structure, the
hydrogen bond layers are closed to form tubes. Due to the different position of the chlorine substituent in
the two compound families, the halogen interactions are oriented towards the inside of the hydrogen-
bonded structures or positioned between the layers and establish a relatively strong connection between
them. The two different halogen positions and every possible combinations of configurations in the six
investigated salts provide a quite detailed landscape of the effect of stereochemistry on the solid-state
structure of the salts. The number of crystal structures of diastereomeric salt pairs and especially of double salts is limited in the
literature. This work exceptionally presents the structures of two constitutional isomer double salts along
with their related diastereomeric salt pairs, which were successfully crystallized and their structures
elucidated presenting two complete sets of chiral systems. These results provide a deeper insight into chiral
recognition and contribute to the mastery of synthon engineering. The investigated systems are
1-cyclohexylethylammonium 2 -chloromandelate ( $S$ - $S, R-S$, SS-SR) and 1-cyclohexylethylammonium
4 -chloromandelate ( $R-R$, $S$ - $R$, SS-SR). The crystal structures and the thermal properties of all diastereomers
(including the less stable diastereomers) and double salts have been determined and comparatively
analysed. In the crystal of five of the six chiral salts, hydrogen bonded layers are formed with the
participation of the ionic groups and the hydroxyl group of the mandelate anion. In one structure, the
hydrogen bond layers are closed to form tubes. Due to the different position of the chlorine substituent in
the two compound families, the halogen interactions are oriented towards the inside of the hydrogen-
bonded structures or positioned between the layers and establish a relatively strong connection between
them. The two different halogen positions and every possible combinations of configurations in the six
investigated salts provide a quite detailed landscape of the effect of stereochemistry on the solid-state
structure of the salts. The number of crystal structures of diastereomeric salt pairs and especially of double salts is limited in the
literature. This work exceptionally presents the structures of two constitutional isomer double salts along
with their related diastereomeric salt pairs, which were successfully crystallized and their structures
elucidated presenting two complete sets of chiral systems. These results provide a deeper insight into chiral
recognition and contribute to the mastery of synthon engineering. The investigated systems are
1-cyclohexylethylammonium 2 -chloromandelate ( $S$ - $S, R-S$, SS-SR) and 1-cyclohexylethylammonium
4 -chloromandelate ( $R-R$, $S$ - $R$, SS-SR). The crystal structures and the thermal properties of all diastereomers
(including the less stable diastereomers) and double salts have been determined and comparatively
analysed. In the crystal of five of the six chiral salts, hydrogen bonded layers are formed with the
participation of the ionic groups and the hydroxyl group of the mandelate anion. In one structure, the
hydrogen bond layers are closed to form tubes. Due to the different position of the chlorine substituent in
the two compound families, the halogen interactions are oriented towards the inside of the hydrogen-
bonded structures or positioned between the layers and establish a relatively strong connection between
them. The two different halogen positions and every possible combinations of configurations in the six
investigated salts provide a quite detailed landscape of the effect of stereochemistry on the solid-state
structure of the salts. The number of crystal structures of diastereomeric salt pairs and especially of double salts is limited in the
literature. This work exceptionally presents the structures of two constitutional isomer double salts along
with their related diastereomeric salt pairs, which were successfully crystallized and their structures
elucidated presenting two complete sets of chiral systems. These results provide a deeper insight into chiral
recognition and contribute to the mastery of synthon engineering. The investigated systems are
1-cyclohexylethylammonium 2 -chloromandelate ( $S$ - $S, R-S$, SS-SR) and 1-cyclohexylethylammonium
4 -chloromandelate ( $R-R$, $S$ - $R$, SS-SR). The crystal structures and the thermal properties of all diastereomers
(including the less stable diastereomers) and double salts have been determined and comparatively
analysed. In the crystal of five of the six chiral salts, hydrogen bonded layers are formed with the
participation of the ionic groups and the hydroxyl group of the mandelate anion. In one structure, the
hydrogen bond layers are closed to form tubes. Due to the different position of the chlorine substituent in
the two compound families, the halogen interactions are oriented towards the inside of the hydrogen-
bonded structures or positioned between the layers and establish a relatively strong connection between
them. The two different halogen positions and every possible combinations of configurations in the six
investigated salts provide a quite detailed landscape of the effect of stereochemistry on the solid-state
structure of the salts. The number of crystal structures of diastereomeric salt pairs and especially of double salts is limited in the
literature. This work exceptionally presents the structures of two constitutional isomer double salts along
with their related diastereomeric salt pairs, which were successfully crystallized and their structures
elucidated presenting two complete sets of chiral systems. These results provide a deeper insight into chiral
recognition and contribute to the mastery of synthon engineering. The investigated systems are
1-cyclohexylethylammonium 2 -chloromandelate ( $S$ - $S, R-S$, SS-SR) and 1-cyclohexylethylammonium
4 -chloromandelate ( $R-R$, $S$ - $R$, SS-SR). The crystal structures and the thermal properties of all diastereomers
(including the less stable diastereomers) and double salts have been determined and comparatively
analysed. In the crystal of five of the six chiral salts, hydrogen bonded layers are formed with the
participation of the ionic groups and the hydroxyl group of the mandelate anion. In one structure, the
hydrogen bond layers are closed to form tubes. Due to the different position of the chlorine substituent in
the two compound families, the halogen interactions are oriented towards the inside of the hydrogen-
bonded structures or positioned between the layers and establish a relatively strong connection between
them. The two different halogen positions and every possible combinations of configurations in the six
investigated salts provide a quite detailed landscape of the effect of stereochemistry on the solid-state
structure of the salts. The number of crystal structures of diastereomeric salt pairs and especially of double salts is limited in the
literature. This work exceptionally presents the structures of two constitutional isomer double salts along
with their related diastereomeric salt pairs, which were successfully crystallized and their structures
elucidated presenting two complete sets of chiral systems. These results provide a deeper insight into chiral
recognition and contribute to the mastery of synthon engineering. The investigated systems are
1-cyclohexylethylammonium 2 -chloromandelate ( $S$ - $S, R-S$, SS-SR) and 1-cyclohexylethylammonium
4 -chloromandelate ( $R-R$, $S$ - $R$, SS-SR). The crystal structures and the thermal properties of all diastereomers
(including the less stable diastereomers) and double salts have been determined and comparatively
analysed. In the crystal of five of the six chiral salts, hydrogen bonded layers are formed with the
participation of the ionic groups and the hydroxyl group of the mandelate anion. In one structure, the
hydrogen bond layers are closed to form tubes. Due to the different position of the chlorine substituent in
the two compound families, the halogen interactions are oriented towards the inside of the hydrogen-
bonded structures or positioned between the layers and establish a relatively strong connection between
them. The two different halogen positions and every possible combinations of configurations in the six
investigated salts provide a quite detailed landscape of the effect of stereochemistry on the solid-state
structure of the salts. The number of crystal structures of diastereomeric salt pairs and especially of double salts is limited in the
literature. This work exceptionally presents the structures of two constitutional isomer double salts along
with their related diastereomeric salt pairs, which were successfully crystallized and their structures
elucidated presenting two complete sets of chiral systems. These results provide a deeper insight into chiral
recognition and contribute to the mastery of synthon engineering. The investigated systems are
1-cyclohexylethylammonium 2 -chloromandelate ( $S$ - $S, R-S$, SS-SR) and 1-cyclohexylethylammonium
4 -chloromandelate ( $R-R$, $S$ - $R$, SS-SR). The crystal structures and the thermal properties of all diastereomers
(including the less stable diastereomers) and double salts have been determined and comparatively
analysed. In the crystal of five of the six chiral salts, hydrogen bonded layers are formed with the
participation of the ionic groups and the hydroxyl group of the mandelate anion. In one structure, the
hydrogen bond layers are closed to form tubes. Due to the different position of the chlorine substituent in
the two compound families, the halogen interactions are oriented towards the inside of the hydrogen-
bonded structures or positioned between the layers and establish a relatively strong connection between
them. The two different halogen positions and every possible combinations of configurations in the six
investigated salts provide a quite detailed landscape of the effect of stereochemistry on the solid-state
structure of the salts. The number of crystal structures of diastereomeric salt pairs and especially of double salts is limited in the
literature. This work exceptionally presents the structures of two constitutional isomer double salts along
with their related diastereomeric salt pairs, which were successfully crystallized and their structures
elucidated presenting two complete sets of chiral systems. These results provide a deeper insight into chiral
recognition and contribute to the mastery of synthon engineering. The investigated systems are
1-cyclohexylethylammonium 2 -chloromandelate ( $S$ - $S, R-S$, SS-SR) and 1-cyclohexylethylammonium
4 -chloromandelate ( $R-R$, $S$ - $R$, SS-SR). The crystal structures and the thermal properties of all diastereomers
(including the less stable diastereomers) and double salts have been determined and comparatively
analysed. In the crystal of five of the six chiral salts, hydrogen bonded layers are formed with the
participation of the ionic groups and the hydroxyl group of the mandelate anion. In one structure, the
hydrogen bond layers are closed to form tubes. Due to the different position of the chlorine substituent in
the two compound families, the halogen interactions are oriented towards the inside of the hydrogen-
bonded structures or positioned between the layers and establish a relatively strong connection between
them. The two different halogen positions and every possible combinations of configurations in the six
investigated salts provide a quite detailed landscape of the effect of stereochemistry on the solid-state
structure of the salts. The number of crystal structures of diastereomeric salt pairs and especially of double salts is limited in the
literature. This work exceptionally presents the structures of two constitutional isomer double salts along
with their related diastereomeric salt pairs, which were successfully crystallized and their structures
elucidated presenting two complete sets of chiral systems. These results provide a deeper insight into chiral
recognition and contribute to the mastery of synthon engineering. The investigated systems are
1-cyclohexylethylammonium 2 -chloromandelate ( $S$ - $S, R-S$, SS-SR) and 1-cyclohexylethylammonium
4 -chloromandelate ( $R-R$, $S$ - $R$, SS-SR). The crystal structures and the thermal properties of all diastereomers
(including the less stable diastereomers) and double salts have been determined and comparatively
analysed. In the crystal of five of the six chiral salts, hydrogen bonded layers are formed with the
participation of the ionic groups and the hydroxyl group of the mandelate anion. In one structure, the
hydrogen bond layers are closed to form tubes. Due to the different position of the chlorine substituent in
the two compound families, the halogen interactions are oriented towards the inside of the hydrogen-
bonded structures or positioned between the layers and establish a relatively strong connection between
them. The two different halogen positions and every possible combinations of configurations in the six
investigated salts provide a quite detailed landscape of the effect of stereochemistry on the solid-state
structure of the salts. The number of crystal structures of diastereomeric salt pairs and especially of double salts is limited in the
literature. This work exceptionally presents the structures of two constitutional isomer double salts along
with their related diastereomeric salt pairs, which were successfully crystallized and their structures
elucidated presenting two complete sets of chiral systems. These results provide a deeper insight into chiral
recognition and contribute to the mastery of synthon engineering. The investigated systems are
1-cyclohexylethylammonium 2 -chloromandelate ( $S$ - $S$, $R-S$, $S S-S R$ ) and 1 -cyclohexylethylammonium
4-chloromandelate ( $R-R, S-R$, SS-SR). The crystal structures and the thermal properties of all diastereomers
(including the less stable diastereomers) and double salts have been determined and comparatively
analysed. In the crystal of five of the six chiral salts, hydrogen bonded layers are formed with the
participation of the ionic groups and the hydroxyl group of the mandelate anion. In one structure, the
hydrogen bond layers are closed to form tubes. Due to the different position of the chlorine substituent in
the two compound families, the halogen interactions are oriented towards the inside of the hydrogen-
bonded structures or positioned between the layers and establish a relatively strong connection between
them. The two different halogen positions and every possible combinations of configurations in the six
investigated salts provide a quite detailed landscape of the effect of stereochemistry on the solid-state
structure of the salts.
\end{abstract}

\section{Introduction}

Optical resolution via diastereomeric salt formation is the most simple and robust and therefore, the most frequently used method for enantiomer purification. However, it is usually not straight forward to find the ideal resolving agent and optical resolution conditions.

In the case of a racemic mixture of enantiomers, the crystalline phase may contain a 1 to 1 mixture of the crystals

\footnotetext{
${ }^{a}$ Chemical Crystallography Research Laboratory, Research Centre for Natural Sciences, Hungary.E-mail: nagyne.bereczki.laura@ttk.hu

${ }^{b}$ Plasma Chemistry Research Group, Institute of Materials and Environmental Chemistry, Research Centre for Natural Sciences, Hungary

${ }^{c}$ Department of Chemical and Environmental Process Engineering, Faculty of Chemical Technology and Biotechnology, Budapest University of Technology and Economics, Budapest, Hungary

${ }^{d}$ Organocatalysis Research Group, Institute of Organic Chemistry, Research Centre for Natural Sciences, Hungarian Academy of Sciences, Budapest, Hungary $\dagger$ Electronic supplementary information (ESI) available. CCDC 2059730-2059735. For ESI and crystallographic data in CIF or other electronic format see DOI: $10.1039 / \mathrm{d} 1 \mathrm{ce} 00145 \mathrm{k}$
}

of the pure enantiomers, that is a conglomerate or the 1 to 1 ratio of the enantiomers in a crystal called a racemate. ${ }^{1-5}$ The formation of the racemate is much more probable than that of the enantiomeric crystals. The pure enantiomers can only crystallize in one of the 65 non-enantiogenic Sohncke space groups which contain only rotations, rototranslations and translations that is a notable restriction. Meanwhile the racemate crystals may have mirror plains or inversion centres which are statistically much preferred during the crystal formation.

In an optical resolution procedure via diastereomeric salt formation an optically active resolving agent is also present beside the racemic mixture of the targeted compound. During the resolution experiment two diastereomeric salts may be formed in different ratios. In addition, by analogy of the above, there is also the possibility to obtain a crystalline salt that contains both enantiomers of the starting compound in 1 to 1 ratio and the resolving agent in optically pure form. Formation of such a so called double salt ${ }^{6-8}$ is not so favoured by symmetry considerations, however other factors may promote its formation. The presence of the double salt during 
the optical resolution process practically sets the enantiomeric excess to zero in the product.

The detection of the double salt is possible using DSC technique by the analysis of the crystals received by the evaporation of the solvent from the resolution mixture. The double salt is an independent compound that has only one sharp individual melting peak on the DSC curve. On the other hand, the 1 to 1 mixture of the crystals of the diastereomers gives two melting peaks on the DSC curve. Powder X-ray diffraction is also a suitable method for the investigation of the $1: 1$ ratio phase. The powder X-ray diffractogram of the $1: 1$ ratio physical mixture of the two diastereomers is the sum of the diffractograms of the diastereomers. In case of the double salt, an entirely different powder diffractogram is measured. Nonetheless, the most unambiguous evidence for the existence of the double salt is when one has its crystal structure in hand.

Racemic 1-cyclohexylethylamine can be successfully resolved with mandelic acid, ${ }^{9} \quad O$-methylmandelic acid with 1-cyclohexylethylamine ${ }^{10}$ and para-chloromandelic acid with phenylethylamine. ${ }^{11,12}$ Therefore, it could be presumed that 1-cyclohexylethylamine would be a suitable resolving agent for the optical resolutions of 2- and 4-chloromandelic acids (the interchange of the resolving agent and the racemic compound leads to a mirrored system and therefore the experimental results are comparable). Both of them are intermediates in the production of important bioactive or medical compounds. 2-Chloromandelic acid is a key intermediate for the antithrombotic agent clopidogrel which is an antiplatelet agent to treat coronary artery and vascular diseases. ${ }^{11-14}$ 4-Chloromandelic acid is an important intermediate in the synthesis of drugs against diabetes and lipid disorders. ${ }^{15}$

We found that practically there was no enantiomer discrimination in neither of the two chloromandelic acid resolutions with 1-cyclohexylethylamine resolving agent. In both cases a double salt at 1 to $1 R$ - and $S$-chloromandelic acid ratio was found. The crystal structures of the diastereomers and the double salts have been determined and the structural data used for the explanation of the crystallization properties of the substances. The number of crystal structures of diastereomeric salt pairs ${ }^{16-18}$ or double salts $^{19}$ is limited in the literature. The extensive structural study of the isomeric 1-cyclohexylethylammonium chloromandelate salts fills a niche in this field and gives a deeper insight in chiral recognition during crystallization.

The directed manipulation of the supramolecular packing architecture, e.g. synthon engineering harmonises electrostatic interactions and spatial requirements as far as it is possible. A chiral centre in a molecule introduces barriers to molecular flexibility, limits the formation of supramolecular interactions of functional groups by spatial restrictions to the given molecular configuration. The origin of the difficulty of the formation of diastereomeric crystals can be traced back to the role of the molecular shape which hampers the formation of structural patterns. In case of the presented 1-cyclohexylethylammonium 2-chloromandelate
$[(S)$-(1-cyclohexylethyl)ammonium

(S)-2-chloromandelate

(HOM-2),

$(R)-(1$-cyclohexylethyl)ammonium

$(S)-2-$

chloromandelate (HET-2) and (S)-(1-cyclohexylethyl) ammonium $(R, S)$-2-chloromandelate (DOB-2)] as well as of 1-cyclohexylethylammonium 4-chloromandelate $\quad[(R)-(1-$ cyclohexylethyl)ammonium (R)-4-chloromandelate (HOM-4), $(S)$-(1-cyclohexylethyl)ammonium $\quad(R)$-4-chloromandelate (HET-4) and (S)-(1-cyclohexylethyl)ammonium $(R, S)$-4chloromandelate (DOB-4)] salts we uniquely have in hand two completed series, the structures of the two diastereomers and the double salt of an organic salt in both cases, thus all the three possible combinations of the molecular configurations were available for the comparison (Scheme 1).

\section{Materials and methods}

\subsection{Materials}

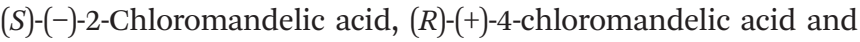
$(R)-(-)$-1-cyclohexylethylamine, $\quad(S)-(+)-1$-cyclohexylethylamine and racemic 1-cyclohexylethylamine and the solvents were purchased from Sigma Aldrich and used without further purification.

Diastereomeric salts were prepared from an equimolar mixture of $60 \mathrm{mg}(S)-(-)-2$-chloromandelic acid and $40 \mathrm{mg}(S)$ $(+)$ - or $(R)-(-)$-1-cyclohexylethylamine and from an equimolar mixture of $60 \mathrm{mg}(R)-(+)-4$-chloromandelic acid and $40 \mathrm{mg}$ $(S)-(+)$ - or $(R)-(-)-1$-cyclohexylethylamine from a saturated dichloromethane solution by precipitation with hexane. The precipitates were filtered out and washed with hexane.

The double salts were crystallized from an equimolar mixture of $60 \mathrm{mg}$ racemic 2 - or 4 -chloromandelic acid and 40 mg (S)-(+)-1-cyclohexylethylamine from a saturated dichloromethane solution by precipitation with hexane. The precipitates were filtered out and washed with hexane.

\subsection{Optical resolution experiments}

Optical resolution experiments were performed under ambient conditions in $5 \mathrm{ml}$ methanol and $5 \mathrm{ml} n$-propanol solvents and in $5 \mathrm{ml} 1: 1$ toluene:acetonitrile solvent mixture. Optical resolution of $100 \mathrm{mg}$ chloromandelic acid was performed with $68 \mathrm{mg}(R)-(-)-1$-cyclohexylethylamine $(0.5$ molar ratio of the resolving agent) in each case. Enantiomeric excess was determined by chiral HPLC.

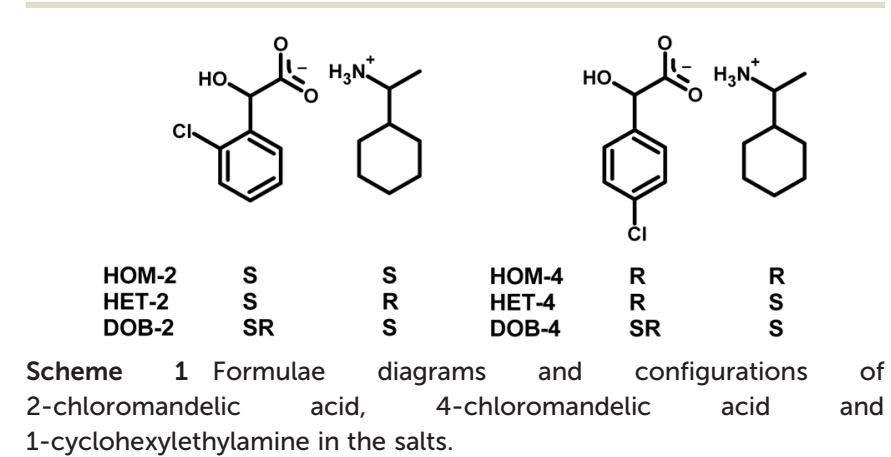

1-cyclohexylethylamine in the salts. 


\subsection{Preparation of the single crystals}

The homochiral diastereomeric salt crystals were harvested from an equimolar mixture of $(S)-(-)-2$-chloromandelic acid and $(S)-(-)$-1-cyclohexylethylamine (HOM-2) and from an equimolar mixture of $(R)-(+)-4$-chloromandelic acid and $(R)$ $(+)$-1-cyclohexylethylamine (HOM-4) from a dichloromethanehexane 1:3 ratio solvent mixture. Heterochiral diastereomeric salts were crystallized from the melt of the mixture of an equimolar mixture of $(S)-(-)$-2-chloromandelic acid and $(R)$ (+)-1-cyclohexylethylamine (HET-2) or $(R)-(+)-4$-chloromandelic acid and $(S)-(-)-1$-cyclohexylethylamine (HET-4). The double salts were crystallized from an equimolar mixture of racemic 2- or 4-chormandelic acid and $(S)-(+)-1$-cyclohexylethylamine (DOB-2 and DOB-4) from methanol solvent.

\subsection{Single crystal X-ray diffraction measurements}

Intensity data were collected on an RAXIS-RAPID II diffractometer $(\mathrm{Cu}$ or Mo sealed X-ray tube radiation source, graphite monochromator, image plate detector). ${ }^{20,21}$ The structures were solved by direct $^{22,23}$ or charge-flipping ${ }^{24}$ methods (and subsequent difference syntheses) and then anisotropic full-matrix least-squares refinement on $F^{2}$ for all non-hydrogen atoms was performed..$^{22,25-30}$ Hydrogen atomic positions were calculated from assumed geometries except some of the hydroxyl hydrogens which were located in difference maps. Hydrogen atoms were included in structure factor calculations but they were not refined. The isotropic displacement parameters of the hydrogen atoms were approximated from the $U(\mathrm{eq})$ value of the atom they were bonded to. Identity of the $\mathrm{CH}_{3}$ and $\mathrm{NH}_{3}$ groups of the cations was established during the refinement on the basis of the hydrogen bond pattern. In most of the cases it was in good agreement with the slightly higher electron density of the nitrogen of the $\mathrm{NH}_{3}$ group. Disordered molecular parts have been restrained in different ways. AFIX 66 was applied to aromatic rings, DFIX to disordered cyclohexyl rings where it was needed. In some cases, in order to smooth the thermal ellipsoids, SIMU, DELU or ISOR restraints were used as well. As it is usual in the case of diastereomers, the crystal qualities vary with the different configurations. Molecular graphics were made by the Mercury software. ${ }^{31-33}$

\subsection{Solubility measurements}

The solubility of the diastereomers and the double salts has been determined in dichloromethane solvent. Dichloromethane was added to 2 to $8 \mathrm{mg}$ of the samples in $20 \mu \mathrm{l}$ portions until the samples dissolved.

\subsection{DSC measurements}

DSC measurements were performed by a METTLER TOLEDO DSC in the $25 \cdots 180{ }^{\circ} \mathrm{C}$ temperature range with $5{ }^{\circ} \mathrm{C} \mathrm{min}^{-1}$ heating rate using 1.0 to $1.2 \mathrm{mg}$ sample in a pierced $40 \mu \mathrm{l}$ alumina crucible in $80 \mathrm{ml} \mathrm{min}{ }^{-1}$ nitrogen atmosphere.

\section{Results and discussion}

\subsection{Optical resolution results}

Cyclohexylethylamine can be successfully resolved with mandelic acid. ${ }^{9}$ In spite of this, in the optical resolution of the halogenated derivatives, 2-chloromandelic acid and 4-chloromandelic acid with 1-cyclohexylethylamine (Table 1) practically zero enantiomeric excess can be obtained. The halogen substitution basically and unexpectedly changes the optical resolution results. The low efficiency of the optical resolutions can be associated with the presence of the double salt during the optical resolution experiments (DOB-2 and DOB-4).

\subsection{Solubility of the diastereomers and the double salts}

The heterochiral and homochiral diastereomers as well as the double salts of both 2-chloromandelic acid and 4-chloromandelic acid have been prepared and their solubility measured in dichloromethane solvent at room temperature. Dichloromethane was selected as a mildly polar solvent because of the large solubility differences. The solubility data are listed in Table 2. The homochiral diastereomers (HOM-2 or HOM-4) have the lower solubility in both systems of the two different diastereomers. This means that in the absence of the double salt, one would have the homochiral diastereomer in the precipitate in the course of an optical resolution experiment. The solubility difference of the homochiral and heterochiral diastereomers is quite pronounced therefore, on the basis of the solubility data, one would expect a good enantiomer separation in the absence of the double salts. The solubilities of the double salts are in both cases lower than the solubility of any of the diastereomers.

What is surprising in the solubility data is that the solubility of HOM-2 and HET-2 is by two orders of magnitude larger than that of the other isomers. All investigated compounds are constitutional or optical isomers with ionic interactions in their solid phase and such a difference in the solubility is not foreseeable. However, these results are in very good agreement with the thermoanalytical data since the melting enthalpy of the same compounds (HOM-2 and HET2) is unusually low (Table 3) taking into account that these are ionic compounds. The trends of the solubility data are in very good agreement with the melting enthalpies especially within one set of compounds. The melting points do not correlate with the solubility data.

Table 1 Optical resolution results of 2-chloromandelic acid with 1-cyclohexylethylamine and 4-chloromandelic acid with 1-cyclohexylethylamine resolving agent in different solvents (enantiomeric excess: ee $=\left|\frac{R-S}{R+S}\right| \times 100 \%$ )

\begin{tabular}{llll}
\hline & 2-Chloromandelic acid & & 4-Chloromandelic acid \\
\cline { 2 - 2 } Solvent & ee\% & ee\% \\
\hline Methanol & 0.0 & 1.8 \\
$n$-Propanol & 3.2 & 1.5 \\
Toluene-ACN 1:1 & 5.7 & 2.2
\end{tabular}


Table 2 Solubility of the diastereomeric and double salts in dichloromethane

\begin{tabular}{lllllll}
\hline Sample & HOM-2 & HET-2 & DOB-2 & HOM-4 & HET-4 & DOB-4 \\
\hline Solubility in DCM mg sample/ml DCM & $300(20)$ & $440(50)$ & $10.4(6)$ & $4.6(2)$ & $9.0(4)$ & $3.2(1)$
\end{tabular}

Table 3 Melting points and enthalpies of the diastereomeric and double salts

\begin{tabular}{lccccc}
\hline Sample & HOM-2 & HET-2 & DOB-2 & HOM-4 & HET-4 \\
\hline Melting point $/{ }^{\circ} \mathrm{C}$ & $137.0(2)$ & $124.8(2)$ & $146.6(2)$ & $156.3(2)$ & $153.0(2)$ \\
Melting enthalpy/kJ per mole & 13.2 & 13.7 & 36.9 & 30.4 & $137.6(2)$ \\
\hline
\end{tabular}

\subsection{Thermal properties and calculated solid-liquid phase diagrams}

The thermal properties were investigated by DSC method (Fig. 1 and 2). In both diastereomer systems, the homochiral diastereomer has the higher melting point as it could be expected being the less soluble diastereomer ${ }^{34-37}$ (Table 3).

The melting enthalpies of HOM-2 and HET-2 are particularly low showing the weakness of the secondary intermolecular interactions in the diastereomeric crystals as compared to the double salt (DOB-2) or to the para-substituted isomers.

The melting point of the less soluble diastereomer (HOM2 and HOM-4) is higher than that of the more soluble diastereomer (HET-2 and HET-4) in both cases, as it was expected. The melting enthalpy of the HOM-2 and HET-2 diastereomers is unusually low which is in good agreement with the outstandingly high solubility of HOM-2 and HET-2.

The melting point of DOB-2 is higher whereas the melting point of DOB-4 is lower than the melting points of the diastereomers. The melting enthalpy of the double salt is considerably higher than that of the related diastereomers in both cases.

\subsection{Analysis of the crystal structures}

Crystal structural and SXRD experimental data are listed in Table S1.†

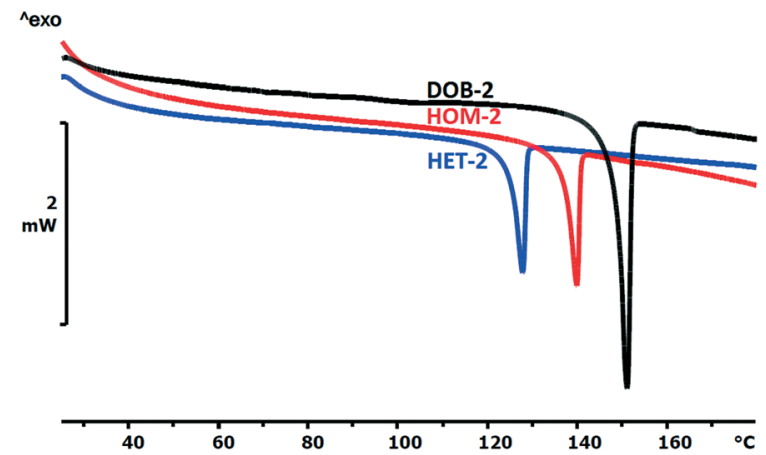

Fig. 1 DSC curves of the diastereomers and double salt of compound 2.
The homochiral and less soluble diastereomers (HOM-2 and HOM-4) crystallize in the orthorhombic crystal system in $P 2_{1} 2_{1} 2_{1}$ space group. The heterochiral diastereomers (HET-2 and HET-4) and the double salts (DOB-2 and DOB-4) crystallize in the lower symmetry monoclinic system in space groups $P 2_{1}$ or $C 2$. HOM-2 has considerably higher crystal density than its optical isomers. The crystal density is not significantly different in case of HOM-4, HET-4 and DOB-4 isomeric salts.

The asymmetric units of the less soluble diastereomers (HOM-2 and HOM-4) contain the minimal one ion pair, $Z^{\prime}=$ 1. On the other hand, the asymmetric units of the more soluble HET-2 and HET-4 contain four ion pairs $\left(Z^{\prime}=4\right)$ (Fig. 3). The molecular geometries of the four different ion pairs are slightly different and in HET-2, the rings are disordered. The multiplication of the ion pairs in the asymmetric unit and the disordered apolar molecular parts reveal difficulties in the crystal packing of the heterochiral diastereomers.

In the case of the double salts, at least two ion pairs are necessarily present in the asymmetric unit, a homochiral and a heterochiral one. In the case of the diastereomers, the crystals are built up of homochiral cations and of homochiral anions. The chirality of the anion and cation can be identical (homochiral diastereomer) or different (heterochiral diastereomer). While in the double salt the anions are present in both configurations. In the crystals of DOB-4, the minimal 2 ion pairs are observed in the asymmetric unit.

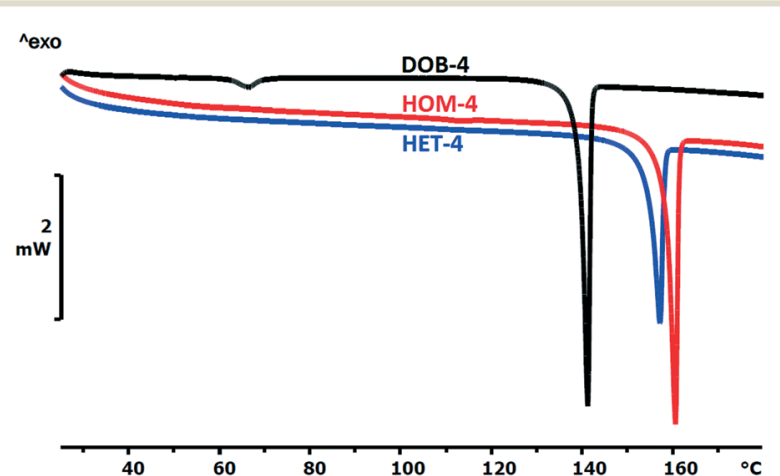

Fig. 2 DSC curves of the diastereomers and double salt of compound 4. 

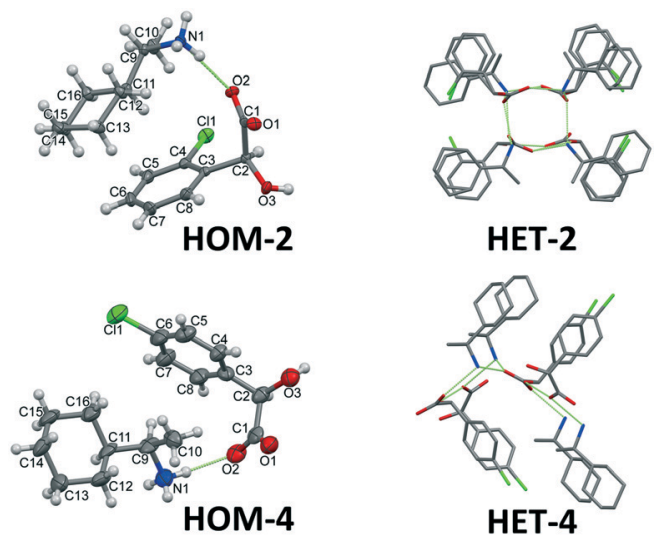

Fig. 3 Asymmetric units of the diastereomeric salts, the asymmetric units of the homochiral HOM-2 and HOM-4 contain one ion pair $\left(Z^{\prime}=\right.$ 1) while the asymmetric units of the heterochiral HET-2 and HET-4 contain four ion pairs $\left(Z^{\prime}=4\right)$ (thermal ellipsoids are represented on the $50 \%$ probability level, where multiple ion pairs are present in the asymmetric unit, stick representation is used and hydrogen atoms are omitted for clarity).

However, in the asymmetric unit of DOB-2, four ion pairs are present (increased $Z^{\prime}$ ) and some of the cyclohexyl rings are disordered (Fig. 4).

In some combinations of the configurations, the crystal packing is less favourable, the positions of the anions and cations may become uncertain (disorder in HET-2 and DOB2) and diversified (increased $Z^{\prime}$ in HET-2, HET-4 and DOB-2) concerning mainly the apolar rings of the ions. Indeed, the crystallization of the heterochiral diastereomers was difficult and their crystals had low rigidity.

\subsection{Comparison of the packing motifs in the isomeric structures}

In the homochiral salts, the anions and the cations are arranged in parallel columns which are parallel to the $a$ crystallographic axis (Fig. 5). The alternating anion and cation columns are connected by electrostatic forces and hydrogen bonds forming 2-dimensional layers in the $a b$ plane.

The packing arrangements of the heterochiral salts are more complicated. In the HET-2 structure, the columns are built up of alternately placed anion-cation pairs. Four
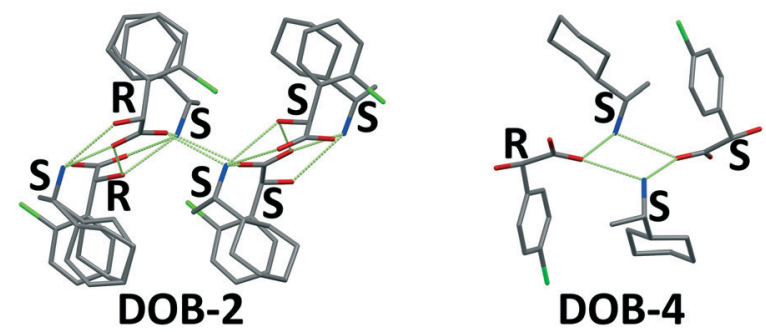

Fig. 4 Asymmetric units of DOB-2 and DOB-4 (chirality of the ion pairs is marked on the figure, hydrogens are omitted for clarity).

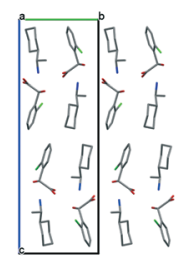

HOM-2

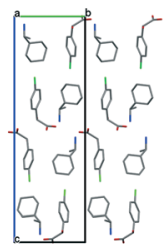

HOM-4

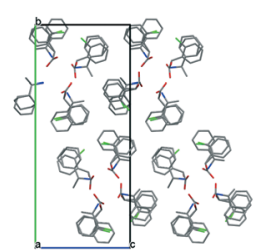

HET-2

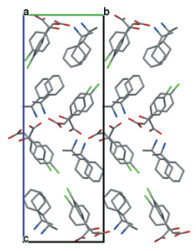

HET-4

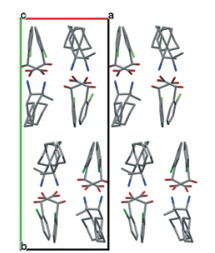

DOB-2

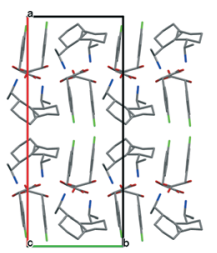

DOB-4
Fig. 5 Comparison of the packing motifs of the salts.

columns are connected together by hydrogen bonds to form one-dimensional hydrogen-bonded units. In the HET-4 structure, similarly to the homochiral structures, anion and cation columns are formed parallel to the $a$ axis and hydrogen bonded 2D layers parallel in the $a b$ plane are also formed. In the case of the heterochiral salts, every second anion and cation have different conformation since HET-2 and HET-4 have four ionpairs in their asymmetric units.

DOB-2 and DOB-4 have similar crystal packings. Here, we can find again the anion and cation columns, which are present as well in the structure of the homochiral salts. In the case of the double salts, the columns run along the $c$ crystallographic axis and hydrogen-bonded planes are formed in DOB-2 in the $a c$ plane and in DOB-4 in the $b c$ plane. Within the columns, every second anion has opposite chirality and every second cation with the same chirality has different conformation. The main difference in the structures is that in DOB-2, two-fold screw axes are running perpendicular to the hydrogen-bonded 2D layers while in DOB-4, two-fold screw axes and two-fold rotation axes are in the $2 \mathrm{D}$ layers or parallel to them, between the layers.

\subsection{Analysis of the hydrogen bonds in the structures, graph set analysis and supramolecular synthons}

The most important structure determining H-bond interactions in these structures are established between the charge-bearing groups of the salts. Owing to their spatial proximity, the hydroxyl groups also take part in these interactions. Characteristic interaction of the carboxylate group in the structures is that they form four strong hydrogen bonds. One of the oxygens binds to two ammonium ions and the other one to one ammonium group and one hydroxyl group of the neighbouring chloromandelate anion (the only exception is HET-4 where the first carboxylate oxygen is in hydrogen bond with one ammonium group but instead of the other ammonium group it binds to a hydroxyl group or forms a $\mathrm{C}-\mathrm{H} \cdots \mathrm{O}$ hydrogen bond). The spatial arrangement of these hydrogen bonds is different depending 
on the chirality of the ions and as a result, they may build up very different hydrogen bond patterns. The hydrogen bond patterns are analysed by graph-set analysis. ${ }^{38,39}$ The hydrogen bonds of the salts are listed in Table S2.†

The arrangement of the ions in the crystal lattice and the hydrogen-bond network is basically changed in the homochiral HOM-2 and HOM-4 salts with the chlorine substitution as compared to the homochiral corresponding non-halogenated derivative $(S)$-1-cyclohexylethylammonium $(S)$-2-chloromandelate $\left(\right.$ QEMZIS $^{9}$ ). QEMZIS has one dimensional hydrogen bonded chains which incorporate only the ionic functional groups of the ions. The complexity of the hydrogen bond pattern is much reduced as compared to the chlorinated derivatives, only $\mathrm{R}_{4}^{2}(8)$ rings (which appear in the double salts as well) and $\mathrm{R}_{2}^{1}(4)$ rings of secondary interactions and intramolecular hydrogen bonds of the hydroxyl group are present (Fig. S1 $\dagger$ ). This polar core is then covered with an apolar shell consisting of phenyl and cyclohexyl rings. In the case of QEMZIS, the hydroxyl group of the mandelic acid does not take part in the main hydrogen bond network of the structure. The cohesion of these one-dimensional units is supported by $\mathrm{C}-\mathrm{H} \cdots \mathrm{O}$ hydrogen bonds between the hydroxyl and cyclohexyl groups and $\mathrm{C}-\mathrm{H} \cdots \pi$ aromatic interactions between the chains. In the case of HOM-2 and HOM-4, the polar groups are arranged in two dimensional layers and the participation of the hydroxyl groups in the hydrogen bond system is increased (Fig. 6). In HOM-2, no notable secondary interactions can be found between the hydrogen bonded layers. In HOM-4, due to the para position of the chlorines, inter-layer $\mathrm{C}-\mathrm{H} \cdots \mathrm{Cl}$ bonds $(\mathrm{C} 14-\mathrm{H} 14 \mathrm{~B} \cdots \mathrm{Cl} 1$, symm. op. 1/2 $x,-y, 1 / 2+z)$ are formed.

In HOM-2, the hydrogen bond network is built up of $\mathrm{R}_{5}^{3}(13), \mathrm{R}_{3}^{2}(9)$ and small $\mathrm{R}_{2}^{1}(4)$ rings (Fig. S2 $\dagger$ ). In HET-2, unlike in any other chlorinated isomers, one-dimensional hydrogen bonded columns are formed. Symmetric $\mathrm{R}_{4}^{4}(12)$ (Fig. S3†) and

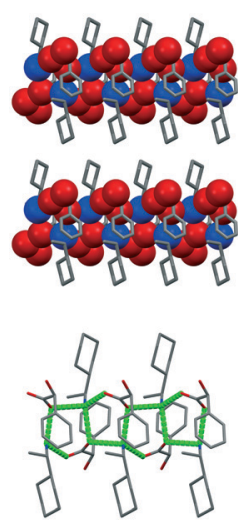

QEMZIS
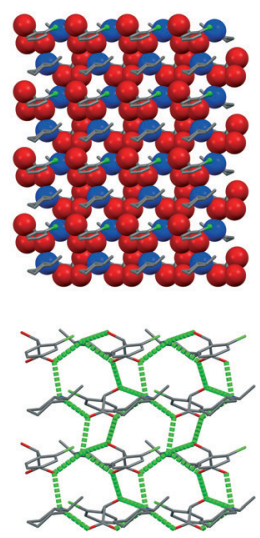

HOM-2
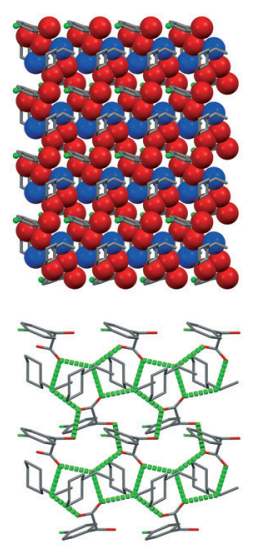

HOM-4
Fig. 6 Hydrogen bond network of the homochiral salts in two different representations: QEMZIS, HOM-2 and HOM-4 (view $c$ axis direction in all cases, nitrogen: space fill, blue; oxygen: space fill, red; carbon: stick, grey; chlorine: ball and stick, green representation, hydrogens omitted for clarity, hydrogen bonds: green).
$\mathrm{R}_{4}^{2}(8)$ rings (Fig. $\mathrm{S} 4 \dagger$ ) connecting two anions and two cations are characteristic patterns and additionally the $R_{2}^{3}(6)$ rings appear. The anions form dimers via $\mathrm{R}_{2}^{2}(10)$ rings (Fig. S3†). In the HOM-4 structure, the main hydrogen bond pattern is again different and consists of $\mathrm{R}_{4}^{3}(10)$ and $\mathrm{R}_{4}^{3}(11)$ rings. Small $\mathrm{R}_{1}^{2}(5)$ rings are also present (Fig. $\mathrm{S} 5 \dagger$ ). In HET-4, the hydrogen bond pattern consists of one large ring, $\mathrm{R}_{5}^{3}(13)$ (similar to HOM-2) and several small and slightly different hydrogen bonded rings $\mathrm{R}_{3}^{2}(7), \mathrm{R}_{3}^{2}(8), \mathrm{R}_{3}^{3}(8), \mathrm{R}_{4}^{3}(8)$ (Fig. S6 $\dagger$ ) as well as $R_{2}^{1}(4)$ and $R_{1}^{2}(5)$ rings similar to those in the other structures.

In the DOB-2 structure, the main characteristics of the hydrogen bond pattern are large $\mathrm{R}_{6}^{4}(18)$ rings (Fig. $\mathrm{S} 7 \dagger$ ) formed with the participation of four anions and two cations. The large rings are connected by $\mathrm{R}_{4}^{2}(8)$ rings including two anions and two cations. The $\mathrm{R}_{1}^{2}(5)$ ring, $\mathrm{R}_{2}^{3}(6)$ ring and $\mathrm{R}_{2}^{1}(4)$ ring mentioned at the diastereomeric salts are also present in this structure. In the DOB-4 structure, a very similar hydrogen bond network is formed that is built up of the same hydrogen bonded rings. The chirality of the DOB-2 and DOB-4 double salts is the same and they form similar hydrogen bond pattern in spite of the different spatial position of the chlorine substituent (ortho or para). In the crystal lattice of the double salts, $R_{6}^{4}(18)$ rings form 2 dimensional honeycomb-like hydrogen bond structures which are very similar for DOB-2 and DOB-4 (Fig. 7).

It can be seen based on the graph-set analysis of the hydrogen bond interactions of the optical isomers that very different patterns are evolved depending on the spatial position of the hydrogen donor and acceptor groups within the ions. The functional groups of the ions are the same in all of the structures however, the hydrogen bond interactions and thus, the supramolecular synthons that are formed in
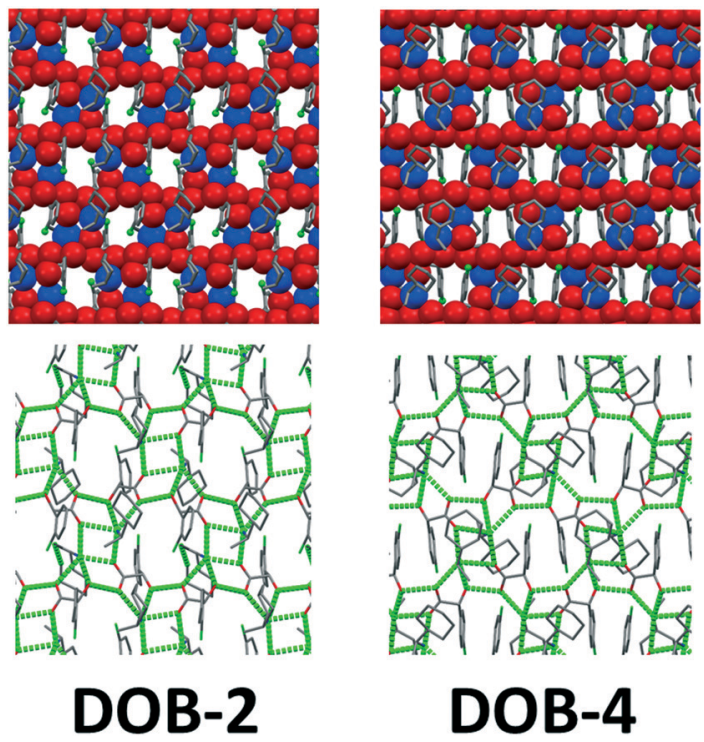

Fig. 7 Similar hydrogen bond system of the double salts DOB-2 and DOB-4 (nitrogen: space fill, blue; oxygen: space fill, red; carbon: stick, grey; chlorine: ball and stick, green representation, hydrogens omitted for clarity). 
the different structures and that can be efficiently described by the different graph-set descriptors, are very diversified depending on the spatial arrangement of the functional groups within the ions.

\subsection{Analysis of the halogen interactions}

In the HOM-2 structure, the ortho chlorine atoms turn in the direction of the main hydrogen bond system in the polar internal part of the molecular layers and take part in it by $\mathrm{Cl} \cdots \mathrm{O}$ interactions established with the alcoholic -OH group (Fig. 8). No chlorine $\cdots$ chlorine interactions can be formed in HOM-2. In the HET-2 structure, intra-column chlorine...chlorine interactions $(\mathrm{Cl} 1 \cdots \mathrm{Cl} 2 \text { 3.468(13) } \AA \text {, 119.9(8) })^{\circ}$ are formed. Between the columns, the cohesion is only set up by apolar interactions. The lack of significant secondary interactions between the hydrogen-bonded units may be responsible for the extremely large solubility and low melting enthalpy of these salts.

In HOM-4, the para-chlorine atoms form chlorine rich layers between the 2D hydrogen bonded polar layers however, chlorine $\cdots$ chlorine interactions are not formed. $\mathrm{C}(14)-\mathrm{H}(14 \mathrm{~A})$

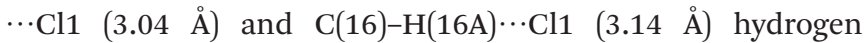
bonds stabilize the layered structure. In HET-4, chlorinechlorine halogen bonds (Cl1A $\cdots$ Cl1D 3.397(6) $\AA$, $168.7^{\circ}$ and Cl1B $\cdots$ Cl1C $\left.3.562(7) \AA, 147.5(5)^{\circ}\right)$ contribute to the stability of the parallel hydrogen bonded layers.

The main difference of the DOB- 2 and DOB-4 double salts can be traced back to the halogen interactions of the orthoand para-chlorine substituent (Fig. 9). In DOB-2, the chlorine atoms turn back to the hydrogen bond system and strengthen it by a $\mathrm{O}(1) \cdots \mathrm{Cl}(1)$ interaction $(\mathrm{O}(1 \mathrm{~B}) \cdots \mathrm{Cl}(1 \mathrm{~A}) \quad 3.193(10) \AA$, $\mathrm{O}(1 \mathrm{C}) \cdots \mathrm{Cl}(1 \mathrm{D}) 3.378(10) \AA$ ) formed with a carboxylate oxygen. In DOB-4, the chlorine atoms form a chlorine rich layer on the outer apolar surfaces of the 2D hydrogen bonded units allowing the formation of $\mathrm{Cl}(1) \cdots \mathrm{Cl}(1)$ halogen bonds (3.341(2) $\AA$, $164.21(18)^{\circ}$ ) between the layers.

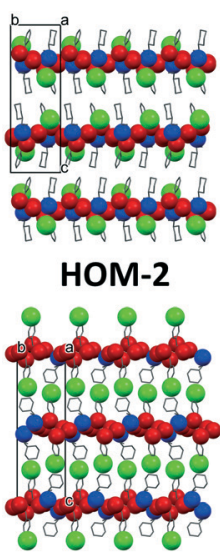

HOM-4

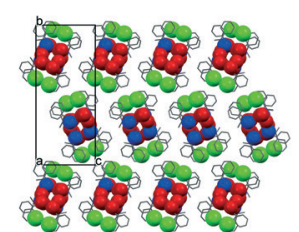

HET-2

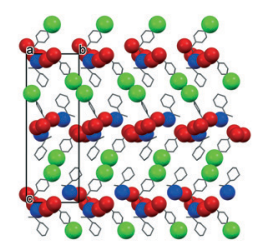

HET-4
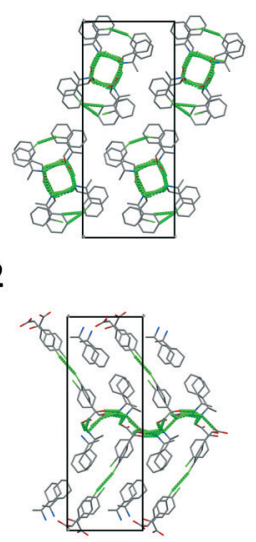

Fig. 8 Chlorine $\cdots$ chlorine interactions in the diastereomers (view from the $a$ axis direction)
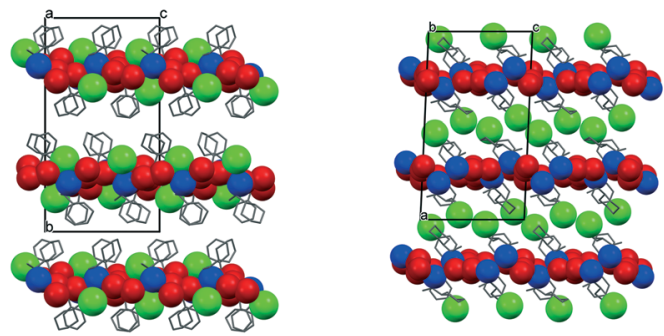

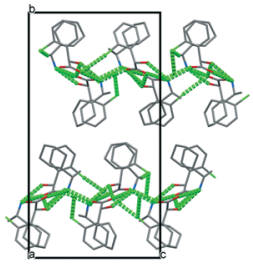

DOB-2

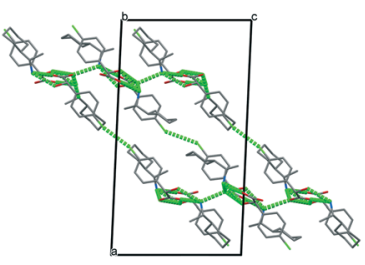

DOB-4
Fig. 9 Halogen interactions of the double salts DOB-2 and DOB-4 (nitrogen: space fill, blue; oxygen: space fill, red; chlorine: space fill, green; carbon: stick representation, hydrogens are omitted for clarity).

\subsection{Comparison of the molecular conformations}

The main torsion angles, namely the torsion of the hydroxyl $\left(\boldsymbol{\Phi}_{1}\right)$ and the carboxylate $\left(\boldsymbol{\Phi}_{2}\right)$ group to the phenyl ring and the torsion of the ammonium group to the cyclohexyl ring $\left(\boldsymbol{\Phi}_{3}\right)$ are listed in Tables 4 (and $\mathrm{S} 2 \dagger$ ). In the homochiral diastreomers (HOM-2 and HOM-4), the selected torsion angles are similar to the analogous dihedral angles of the basic compound QEMZIS (the opposite sign of the torsion angles arises from the different chirality of the ions). In the

Table 4 Comparison of the torsion angles of the anions and cations in the different optical isomers

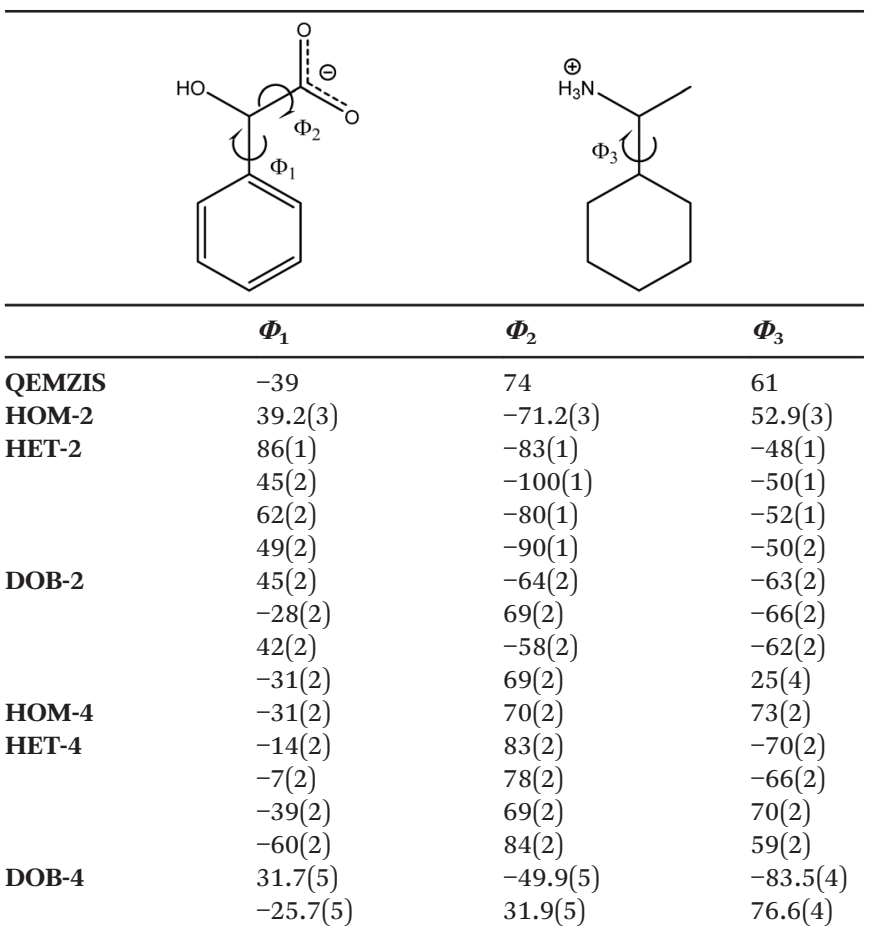




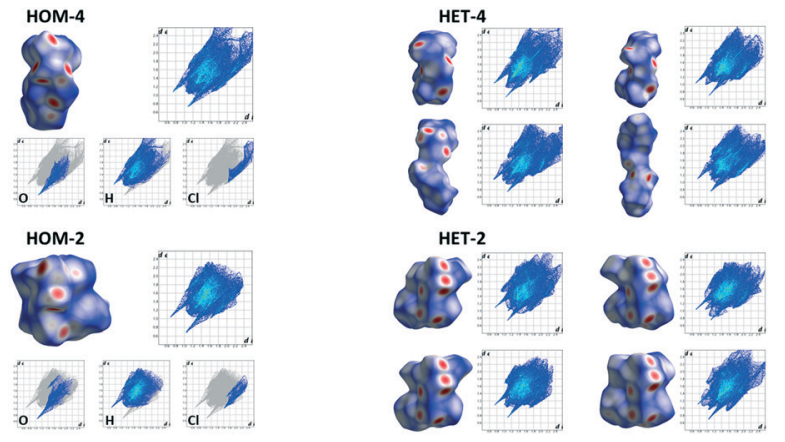

Fig. 10 Hirshfeld surfaces and fingerprint plots calculated for ion pairs in the diastereomeric salts (the Hirshfeld surfaces were mapped over $d_{\text {norm }}$ which is the contact distance of the atoms normalised by their van der Waals radii, calculated by Crystal Explorer software, ${ }^{40}$ the ionpairs are represented in the way that the carboxylate and ammonium moieties are placed in the same position).

case of the optical isomers (HET-2, HET-4), notable differences can be seen in the geometries of the ions within the asymmetric unit. When the asymmetric unit contains several ion pairs, the conformations of the anions or cations are diverse (DOB-2 cation, HET-4 anion) and large differences can be observed in the torsion angles.

\subsection{Hirshfeld analysis of the salts}

Hirshfeld surface analysis is a descriptive graphical tool which helps to elucidate differences in the packing of similar molecules (e.g. polymorphs, isomers or derivatives). Hirshfeld surfaces were calculated (Fig. 10) for the ionpairs of the different diastereomeric salts (HOM-2 and HET-2, HOM-4 and HET-4). Ion pairs were chosen in the way that the interactions between the anion and cation were maximized. For a given anion, the cation whose ammonium group is the closest to the carboxylate group of the anion was chosen. In this case, the ammonium group forms hydrogen bond interaction with one or two oxygens of the carboxylate group. In the structures of the homochiral diastereomers, only one ion pair can be found in the asymmetric unit. In the case of the heterochiral diastereomers, four ionpairs are in the asymmetric unit and the Hirshfeld surfaces have been calculated for each of the four different ionpairs. In HET-4, two types of ionpairs can be found. The Hirshfeld surfaces of
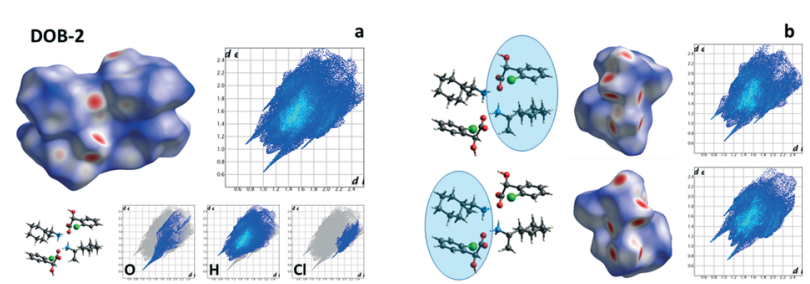

Fig. 11 Hirshfeld surfaces and fingerprint plots for two ionpairs of DOB-2 together (a) and for two different ionpairs separately (b) (the Hirshfeld surfaces were mapped over $d_{\text {norm }}$ which is the contact distance of the atoms normalised by their van der Waals radii).
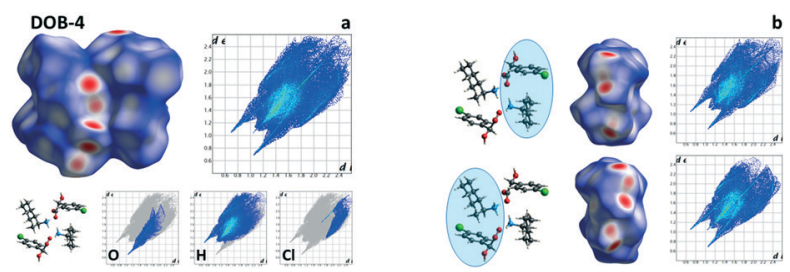

Fig. 12 Hirshfeld surfaces and fingerprint plots calculated for the asymmetric unit of DOB-4 (a) and for the two different ionpairs in the asymmetric unit (b) (the Hirshfeld surfaces were mapped over $d_{\text {norm }}$ which is the contact distance of the atoms normalised by their van der Waals radii).

two of the ionpairs are similar to that of HOM-4 however, less close interactions can be observed on the surfaces. The shapes and the Hirshfeld surfaces of the other two ionpairs are different from HOM-4. With the aid of Hirshfeld surface analysis, significant differences can be observed even in the distances of the strong electrostatic and hydrogen bond interactions of the ionpairs of the para-chloro isomer.

In HET-2, the spatial positions of the intermolecular interactions showed by the Hirshfeld surfaces are rather similar in the cases of the four ionpairs however, differences can be observed in the shapes of the surfaces. In HET-2, the spatial arrangements of the strong interactions remain similar in all four ionpairs, the differences show up mainly in the position of the apolar molecular moieties. In HET-2 and HOM-2, the steric arrangements of the strong interactions are quite different.

In case of DOB-2 and DOB-4, Hirshfeld surfaces have been calculated for a homochiral and a heterochiral ionpair in each structure (Fig. 11b and 12b) and additionally for the two ionpairs together (Fig. 11a and 12b). The positions of the close contacts showed by the Hirshfeld surfaces in the case of the two double salts are quite similar which could be expected on the basis of the very similar hydrogen bonded systems. However, there are some slight differences. The fingerprint plots are much more symmetric in DOB-4, the internal $\left(d_{\mathrm{i}}\right)$ and external $\left(d_{\mathrm{e}}\right)$ distances are similar whereas in DOB-2, marked differences can be observed between these values. In DOB-4, the light blue regions in the fingerprint plots are more marked than in DOB-2 thus, the number of short interactions is higher in the DOB-4 structure.

In both structures, the Hirshfeld surfaces of the two diastereomeric ionpairs are nearly mirror images of each other. Their interactions in the double salts are not similar to those of the ionpairs in the diastereomeric salts.

Properties of the Hirshfeld surfaces of the optical isomers (Table $\mathrm{S} 3 \dagger$ ) and the percentage of the total Hirshfeld surface area of the short interactions of the different atom types (Table S4) are listed in the ESI. $\dagger$

\section{Conclusions}

The investigated systems of 1-cyclohexylethylammonium 2-chloromandelate $(S-S, \quad R-S, \quad S S-S R) \quad$ and 
1-cyclohexylethylammonium 4-chloromandelate $(R-R, S-R$, $S S-S R$ ) are two complete sets of a double salt and related diastereomeric salt pairs, which were successfully crystallized, and their structures determined. It has provided insight into chiral recognition processes.

The halogen substitution has unexpectedly and ultimately changed the outcome of the optical resolution. The ortho-chloro substitution strengthen the polar region of the crystal lattice with its interactions. The para-chloro substitution makes possible to form halogen bonds between the apolar layers of the crystal lattice. The different chirality of the molecules and thus the different molecular shapes directly influence the system of secondary interactions and the interaction strengths in the crystal lattice. These differences then appear on the macroscopic level as well. Depending on the position of the chlorine substituent, large difference in the solubility and melting enthalpy data is experienced.

Hirshfeld surface analysis was used to get a graphic description of the molecular shapes and the strength and spatial arrangement of the intramolecular interactions.

Halogen substitution of a chiral compound, and thus the possibility of the formation of halogen bonds in the crystal lattice import a determining factor in the crystal formation during optical resolution via diastereomeric salt formation. The halogen interactions overwrite even the main hydrogen bonding pattern and a basically different structure may be produced. It can be deduced, that in the case of halogenated compounds, the selection of the resolving agent should not be carried out solely based on hydrogen bonding considerations.

The supramolecular synthons in the case of the salts with the same constitution but variable configuration are the same. In the two diastereomeric and the double salt, all possible mutual spatial arrangement of the synthons is realised. The different spatial arrangement of the synthons leads to the realization of different solid phase properties. The diversity of the isomeric structures comes from the different ability of the given combination of mirror image molecules to form strong intermolecular interactions and close packed structures. In the case of some combinations of the configurations, the crystal formation is hampered and disordered molecular fragments or multiple ion pairs appear in the asymmetric unit.

Structure-property relationship could be established in the two sets of compounds. The structural analyses correspond well with thermal and solubility properties. The structural features and supramolecular interactions reveal the reason of the poor enantiomer discrimination in the optical resolution experiments and differences in macroscopic properties, like solubility, melting temperature and enthalpy of the salts.

\section{Conflicts of interest}

There are no conflicts to declare.

\section{Acknowledgements}

This work was supported by the National Research, Development and Innovation Office-NKFIH through OTKA K124544, KH129588 and PD128504.

\section{References}

1 J. von Langermann, E. Temmel, A. Seidel-Morgenstern and H. Lorenz, J. Chem. Eng. Data, 2015, 60, 721-728.

2 G. Coquerel, in Novel Optical Resolution Technologies, ed. K. Sakai, N. Hirayama and R. Tamura, Springer, Berlin, Heidelberg, 2007, pp. 1-51.

3 S. Srisanga and J. H. ter Horst, Cryst. Growth Des., 2010, 10, 1808-1812.

4 R. J. Davey, G. Sadiq, K. Back, L. Wilkinson and C. C. Seaton, Chem. Commun., 2012, 48, 1976-1978.

5 S. J. Coles, A. L. Ellis, K. Leung, J. Sarson, T. L. Threlfall and G. J. Tizzard, CrystEngComm, 2014, 16, 10816-10823.

6 A. Białońska and Z. Ciunik, Cryst. Growth Des., 2013, 13, 111-120.

7 E. Ganczar, A. Białońska and Z. Ciunik, Cryst. Growth Des., 2019, 19, 4632-4640.

8 P. Marchand, L. Lefèbvre, F. Querniard, P. Cardinaël, G. Perez, J.-J. Counioux and G. Coquerel, Tetrahedron: Asymmetry, 2004, 15, 2455-2465.

9 K. Sakai, R. Sakurai and N. Hirayama, Tetrahedron: Asymmetry, 2006, 17, 1812-1816.

10 A. D. Zodge, P. Bombicz, E. Székely, G. Pokol and J. Madarász, Thermochim. Acta, 2017, 648, 23-31.

11 Q. He, Y.-F. Peng and S. Rohani, Chirality, 2010, 22, 16-23.

12 Q. He, S. Rohani, J. Zhu and H. Gomaa, Cryst. Growth Des., 2010, 10, 5136-5145.

13 S. E. Gilks, R. J. Davey, R. K. Mughal, G. Sadiq and L. Black, Cryst. Growth Des., 2013, 13, 4323-4329.

14 L. M. van Langen, F. van Rantwijk and R. A. Sheldon, Org. Process Res. Dev., 2003, 7, 828-831.

15 A. D. Adams, A. B. Jones, J. P. Berger, J. F. Dropinski, A. Elbrecht, K. Liu, K. L. Macnaul, G.-Q. Shi, J. Langen and G. Zhou, WO2002064094A2, World Intellectual Property Organization, 2002.

16 A. Langkilde, J. Oddershede and S. Larsen, Acta Crystallogr., Sect. B: Struct. Sci., 2002, 58, 1044-1050.

17 R. Yoshioka, H. Hiramatsu, K. Okamura, I. Tsujioka and S. Yamada, J. Chem. Soc., Perkin Trans. 2, 2000, 2121-2128.

18 O. Sánchez-Guadarrama, F. Mendoza-Navarro, A. CedilloCruz, H. Jung-Cook, J. I. Arenas-García, A. Delgado-Díaz, D. Herrera-Ruiz, H. Morales-Rojas and H. Höpfl, Cryst. Growth Des., 2016, 16, 307-314.

19 O. M. Peeters, N. M. Blaton and C. J. De Ranter, Acta Crystallogr., Sect. C: Cryst. Struct. Commun., 1997, 53, 597-599.

20 CrystalClear SM 1.4.0, Rigaku/MSC Inc., 2008.

21 T. Higashi, NUMABS, rev. 2002, Rigaku/MSC Inc., 1998.

22 G. M. Sheldrick, Acta Crystallogr., Sect. A: Found. Crystallogr., 2008, 64, 112-122. 
23 M. C. Burla, R. Caliandro, M. Camalli, B. Carrozzini, G. L. Cascarano, L. De Caro, C. Giacovazzo, G. Polidori and R. Spagna, J. Appl. Crystallogr., 2005, 38, 381-388.

24 L. Palatinus and G. Chapuis, J. Appl. Crystallogr., 2007, 40, 786-790.

25 SHELXL and G. M. Sheldrick, SHELXL-2013 Program for Crystal Structure Solution, University of Göttingen, Germany, 2013.

26 L. J. Farrugia, J. Appl. Crystallogr., 2012, 45, 849-854.

27 L. J. Barbour, J. Supramol. Chem., 2001, 1, 189-191.

28 A. J. C. Wilson, International Tables for X-ray Crystallography, Kluwer Academic Publishers, 1992, vol. C.

29 H. D. Flack, Acta Crystallogr., Sect. A: Found. Crystallogr., 1983, 39, 876-881.

30 S. Parsons and H. Flack, Acta Crystallogr., Sect. A: Found. Crystallogr., 2004, 60, s61.

31 A. L. Spek, J. Appl. Crystallogr., 2003, 36, 7-13.
32 L. J. Farrugia, PLATON, Windows implementation, Dept. of Chemistry, University of Glasgow, UK, 1998.

33 C. F. Macrae, P. R. Edgington, P. McCabe, E. Pidcock, G. P. Shields, R. Taylor, M. Towler and J. van de Streek, J. Appl. Crystallogr., 2006, 39, 453-457.

34 D. Ager, Handbook of Chiral Chemicals, CRC Press, 2005.

35 D. Kozma, G. Pokol and M. Ács, J. Chem. Soc., Perkin Trans. 2, 1992, 435-439.

36 D. Kozma, CRC Handbook of Optical Resolutions via Diastereomeric Salt Formation, CRC Press, 2001.

37 A. Ohta, Y. Hata, Y. Mizuno, T. Asakawa and S. Miyagishi, J. Phys. Chem. B, 2004, 108, 12204-12209.

38 M. C. Etter, J. C. MacDonald and J. Bernstein, Acta Crystallogr., Sect. B: Struct. Sci., 1990, 46, 256-262.

39 J. Bernstein, R. E. Davis, L. Shimoni and N.-L. Chang, Angew. Chem., Int. Ed. Engl., 1995, 34, 1555-1573.

40 M. A. Spackman and D. Jayatilaka, CrystEngComm, 2009, 11, 19-32. 